\title{
The Generalization of Prime Modules
}

\author{
M. Gurabi \\ Department of Mathematical Sciences, Isfahan University of Technology, Isfahan 84156-83111, Iran
}

Correspondence should be addressed to M. Gurabi; m_gurabi@math.iut.ac.ir

Received 29 December 2012; Accepted 15 February 2013

Academic Editor: Masoud Hajarian

Copyright (C) 2013 M. Gurabi. This is an open access article distributed under the Creative Commons Attribution License, which permits unrestricted use, distribution, and reproduction in any medium, provided the original work is properly cited.

Piecewise prime (PWP) module $M_{R}$ is defined in terms of a set of triangulating idempotents in $R$. The class of PWP modules properly contains the class of prime modules. Some properties of these modules are investigated here.

\section{Introduction}

All rings are associative, and $R$ denotes a ring with unity 1 . The word ideal without the adjective right or left means twosided ideal. The right annihilator of ideals of $R$ is denoted by $\operatorname{r.ann}_{R}(I)$. A ring $R$ is quasi-Baer (Baer) if the right annihilator of every right ideal (nonempty subset) of $R$ is generated as a right ideal by an idempotent. We now recall a few definitions and results from [1] which motivated our study and serve as the background material for the present work. An idempotent $e \in R$ is a left semicentral idempotent if exe $=x e$, for all $x \in R$. Similarly right semicentral idempotent can be defined. The set of all left (right) semicentral idempotents of $R$ is denoted by $S_{l}(R)\left(S_{r}(R)\right)$. An idempotent $e \in R$ is semicentral reduced if $S_{l}(e R e)=\{0, e\}$. If 1 is semicentral reduced, then $R$ is called semicentral reduced. An ordered set $\left\{e_{1}, \ldots, e_{n}\right\}$ of nonzero distinct idempotents of $R$ is called a set of left triangulating idempotents of $R$ if all the following hold:

(i) $e_{1}+\cdots+e_{n}=1$,

(ii) $e_{1} \in S_{l}(R)$,

(iii) $e_{k+1} \in S_{l}\left(c_{k} R c_{k}\right)$, where $c_{k}=1-\left(e_{1}+\cdots+e_{k}\right)$ for $1 \leq k \leq n$

From part (iii) of the previous definition, it can be seen that a set of left triangulating idempotents is a set of pairwise orthogonal idempotents. A set $E=\left\{e_{1}, \ldots, e_{n}\right\}$ of left triangulating idempotents of $R$ is complete, if each $e_{i}$ is semicentral reduced. A (complete) set of right triangulating idempotents is defined similarly. The cardinalities of complete sets of left triangulating idempotents of $R$ are the same and are denoted by $\tau \operatorname{dim}(R)$ [1, Theorem 2.10]. A ring $R$ is called piecewise prime if there exists a complete set of left triangulating idempotents $E=\left\{e_{1}, \ldots, e_{n}\right\}$ of $R$, such that $x R y=0$ implies $x=0$ or $y=0$ where $x \in e_{i} R e_{j}$ and $y \in e_{j} R e_{k}$ for $1 \leq i, j, k \leq n$. In view of this definition we say a proper ideal $I$ in $R$ is a PWP ideal if there is a complete set of left triangulating idempotents $E=\left\{e_{1}, \ldots, e_{n}\right\}$, such that $x R y \subseteq I$ implies $x \in I$ or $y \in I$, where $x \in e_{i} R e_{j}$ and $y \in e_{j} R e_{k}$ for $1 \leq i, j, k \leq n$. If $R$ is PWP, then it is PWP with respect to any complete set of left triangulating idempotents of $R$; furthermore for a ring $R$ with finite $\tau \operatorname{dim}(R), R$ is PWP if and only if $R$ is quasi-Baer [1, Theorem 4.11].

A nonzero right $R$-module $M$ is called a prime module if for any nonzero submodule $N$ of $M, \operatorname{r} \cdot \operatorname{ann}_{R}(N)=r \cdot \operatorname{ann}_{R}(M)$, and a proper submodule $P$ of $M$ is a prime submodule of $M$ if the quotient module $M / P$ is a prime module. The notion of prime submodule was first introduced in $[2,3]$; see also $[4,5]$. It is easy to see that $M$ is a prime $R$-module if and only if for any $m \in M$, and $b \in R$ if $m R b=0$, then $m=0$ or $M b=0$.

In this work the concept of prime modules is developed to piecewise prime modules as it is done for rings in [1]. Throughout this work it is considered that $\tau \operatorname{dim}(R)$ is finite.

\section{Main Results}

Definition 1. Let $M$ be an $R$-module and $S=\operatorname{End}_{R}(M)$.

(1) $M$ is a piecewise prime (PWP) $R$-module with respect to a complete set of left triangulating idempotents $E=$ $\left\{e_{1}, \ldots, e_{n}\right\}$ of $R$, if for any $m \in M, e_{i} \in E$, and $b \in R$,

$$
m e_{i} R_{i} b=0 \Longrightarrow m e_{i}=0 \quad \text { or } M e_{i} b=0
$$


(2) Let $N$ be a submodule of $M$. Then $N$ is a piecewise prime submodule of $M$ with respect to $E$ if $M / N$ is a PWP module with respect to $E$.

(3) $M$ is piecewise endoprime (PWEP) with respect to a complete set of left triangulating idempotents $F=$ $\left\{b_{1}, \ldots, b_{m}\right\}$ of $S$, such that for each nonzero submodule $N \subseteq M, f \in S$, and $b_{i} \in F$, if $f b_{i} N=0$, then $f b_{i}=0$.

By Definition 1, $N$ is a piecewise prime submodule of $M$ with respect to a set of left triangulating idempotents $E$ if for any $m \in M, e_{i} \in E$, and $b \in R$,

$$
m e_{i} R e_{i} b \subseteq N \Longrightarrow m e_{i} \in N \quad \text { or } M e_{i} b \subseteq N .
$$

Example 2. Let $E=\left\{e_{1}, \ldots, e_{n}\right\}$ be a complete set of left triangulating idempotents o $R$.

(1) Let $k_{1}$ and $k_{2}$ be two fields and $R=k_{1} \times k_{2}$. Then $M=R / k_{1} \oplus R / k_{2}$ is not a prime module, but it is piecewise prime with respect to $\{(1,0),(0,1)\}$.

(2) If $M$ is a prime $R$-module, then it is piecewise prime with respect to any set of left triangulating idempotents of $R$.

(3) Homomorphic image of $M_{R}$ needs to be PWP with respect to $E$. For example, $\mathbb{Z}_{\mathbb{Z}}$ is a PWP module with respect to $\{0,1\}$, but $\mathbb{Z}_{4}$ is not PWP because $\operatorname{r.ann}_{r}(\overline{2}) \neq \operatorname{r.ann}_{r}\left(\mathbb{Z}_{4}\right)$.

Corollary 3. If $M$ is a PWP R-module with respect to $E$, then any submodule of $M$ is $P W P$ with respect to $E$.

Proof. It can be seen by Definition 1 .

Proposition 4. Let $R$ be a ring with finite triangulating dimension.

(1) $I$ is a PWP ideal of $R$ if and only if $R / I$ is a PWP $R$ module.

(2) $R$ is a PWP ring if and only if $R_{R}$ is $P W P$.

Proof. The part one is obtained by Definition 1, and for second let $I=0$ in part one.

Proposition 5. Let $M$ be an $R$-module, and let $E=\left\{e_{1}, \ldots, e_{n}\right\}$ be a set of left triangulating idempotents of $R$. Then the following statements are equivalent:

(1) $M$ is PWP with respect to $E$;

(2) for each $N \subseteq M$, ideal $I$ in $R$, and $e_{i} \in E$ if $N e_{i} I=0$ then $\mathrm{Ne}_{i}=0$ or $M e_{i} I=0$;

(3) for each $(m) \subseteq M$, ideal (a) in $R$, and $e_{i} \in E$ if $(m) e_{i}(a)=0$ then $(m) e_{i}=0$ or $M e_{i}(a)=0$.

Proof. (1) $\Rightarrow$ (2) If $N e_{i} \neq 0$, then there exists $n \in N$, such that $n e_{i} \neq 0$, and for any $b \in I, n e_{i} R e_{i} b=0$. By Definition 1 , for each $b \in I, M e_{i} b=0$. This implies that $M e_{i} I=0$.

$(2) \Rightarrow(3)$ In (2), let $N=(m)$ and $I=(a)$.

(3) $\Rightarrow$ (1) Let $m e_{i} R e_{i} b=0$ where $m \in M, e_{i} \in E$, and $b \in R$. Thus $m e_{i} R_{i} R e_{i} b R=0$ or $\left(m e_{i}\right) e_{i}\left(e_{i} b\right)=0$. By (3), $\left(m e_{i}\right) e_{i}=0$ or $M e_{i}\left(e_{i} b\right)=0$. This implies that $m e_{i}=0$ or $M e_{i} b=0$.

Proposition 6. Let $M$ be an $R$-module, $S=\operatorname{End}_{R}(M)$, let $E=$ $\left\{e_{1}, \ldots, e_{n}\right\}$ be a complete set of left triangulating idempotents of $R$, and let $F=\left\{b_{1}, \ldots, b_{m}\right\}$ be a complete set of left triangulating idempotents of $S$.

(1) $M$ is a PWP R-module with respect to $E$ if and only if for each $N \subseteq M$ with $N e_{i} \neq 0$, ann $\left(N e_{i}\right)=\operatorname{ann}_{r}\left(M e_{i}\right)$.

(2) If $M_{R}$ is PWP R-module with respect to $E$, then ann $_{r}(M)$ is a PWP ideal of $R$ with respect to $E$.

(3) If $M_{R}$ is PWEP with respect to $F$ and retractable, then ann $_{r}(M)$ is a PWP ideal of $R$ with respect to $E$.

Proof. (1) If $b \in \operatorname{ann}_{r}\left(N e_{i}\right)$, then there exists $n \in N$, such that $n e_{i} R e_{i} \neq 0$ and $n e_{i} R e_{i} b=0$. Since $M$ is PWP $R$-module with respect to $E$ by Definition $1, M e_{i} b=0$. Hence $\operatorname{ann}_{r}\left(N e_{i}\right)=$ $\operatorname{ann}_{r}\left(M e_{i}\right)$. Conversely let $m e_{i} R e_{i} b=0$ where $e_{i} \in E, m \in M$, $b \in R$, and $m e_{i} \neq 0$. Thus $b \in \operatorname{ann}_{r}\left(\left(m e_{i} R\right) e_{i}\right)$ which means $b \in \operatorname{ann}_{r}\left(M e_{i}\right)$ or $M e_{i} b=0$.

(2) Let $I e_{i} J \subseteq \operatorname{ann}_{r}(M)$ and $I e_{i} \nsubseteq \operatorname{ann}_{r}(M)$. Since $\left(M I e_{i}\right) e_{i} J=0$, and $M$ is a PWP $R$-module with respect to $E$, by Proposition $5, M e_{i} J=0$. Thus $e_{i} J \subseteq \operatorname{ann}_{r}(M)$. This implies that $\operatorname{ann}_{r}(M)$ is a PWP ideal of $R$ with respect to $E$.

(3) Let $I e_{i} J \subseteq \operatorname{ann}_{r}(M)$ where $I e_{i}, e_{i} J \nsubseteq \operatorname{ann}_{r}(M)$. Since $M$ is retractable, then there exists a nonzero homomorphism $f: M \rightarrow M I e_{i}$. There exists $b_{j} \in F$, such that $f b_{j} \neq 0$. Since $I e_{i} J \subseteq \operatorname{ann}_{r}(M), f b_{j} M e_{i} J=0$. By assumption $M$ is PWEP with respect to $F$. This implies that $f b_{j}=0$ which is a contradiction. Hence $\operatorname{ann}_{r}(M)$ is a PWP ideal of $R$ with respect to $E$.

A module $M_{R}$ is called retractable if for any nonzero submodule $N$ of $M, \operatorname{Hom}_{R}(M, N) \neq 0$.

Theorem 7. Let $M$ be an R-module, $S=\operatorname{End}_{R}(M)$, and let $F=\left\{b_{1}, \ldots, b_{m}\right\}$ be a complete set of left triangulating idempotents of $S$.

(1) If ${ }_{S} M$ is a PWP module with respect to $F$, then $S$ is a $P W P$ ring. The converse is true when $M_{R}$ is retractable.

(2) ${ }_{S} M$ is a PWP module with respect to $F$, if and only if $M_{R}$ is PWEP with respect to $F$.

Proof. (1) Let $f b_{i} S b_{i} g=0$ where $f, g \in S, b_{i} \in F$ and $b_{i} g \neq 0$. Thus there exists $m \in M$, such that $b_{i} g m \neq 0$ and $f b_{i} S b_{i} g m=$ 0 . Since ${ }_{S} M$ is PWP with respect to $F, f b_{i} M=0$ which means $f b_{i}=0$. Conversely let $f b_{i} S b_{i} m=0$ and $b_{i} m \neq 0$. Since $M_{R}$ is retractable, there exists a nonzero homomorphism $b_{i} g \in$ $\operatorname{Hom}_{R}\left(M, b_{i} m R\right)$. Thus $f b_{i} S b_{i} g=0$. Since $S$ is PWP, $f b_{i}=0$.

(2) Assume $M$ is a PWP $S$-module with respect to $F$. Let $N \subseteq M$ and $f b_{i} N=0$ where $f \in S$ and $b_{i} \in F$. Since ${ }_{S} M$ is PWP, by Proposition 6(1), $f b_{i} M=0$. Thus $f b_{i}=$ 0 . Conversely assume $M_{R}$ be PWEP with respect to $F$. Let $f b_{i} S b_{i} m=0$ where $f \in S, b_{i} \in F, m \in M$, and $b_{i} m \neq 0$. If $N=S b_{i} m$, then $f b_{i} N=0$. This implies that $f b_{i}=0$ or $f b_{i} M=0$. Hence ${ }_{S} M$ is PWP with respect to $F$. 
Let $M$ be a right $R$-module with $S=\operatorname{End}_{R}(M)$. Then $M_{R}$ is called a quasi-Baer module, if for any $N \subseteq_{S} M, \operatorname{lann}_{S}(N)=$ Se, where $e=e^{2} \in S[6]$.

Corollary 8. Let $M$ be a retractable $R$-module, $S=\operatorname{End}_{R}(M)$, and let $F=\left\{b_{1}, \ldots, b_{m}\right\}$ be a complete set of left triangulating idempotents of $S$. Then the following statements are equivalent:

(1) $M_{R}$ is a PWEP module with respect to $F$;

(2) ${ }_{S} M$ is a PWP module with respect to F;

(3) $M_{R}$ is quasi-Baer.

Proof. (1) $\Leftrightarrow$ (2) This is evident by Theorem 7(2).

(2) $\Leftrightarrow$ (3) By [6, Proposition 4.7], $M_{R}$ is quasi-Baer if and only if $S$ is quasi-Baer. By [1, Theorem 4.11], $S$ is PWP with respect to $F$ if and only if $S$ is quasi-Baer. The result is obtained by Theorem 7(1).

Proposition 9. Let $\Lambda$ be an index set, and let $E=\left\{e_{1}, \ldots, e_{n}\right\}$ be a complete set of left triangulating idempotents of $R$.

(1) Let $M=\oplus_{\lambda \in \Lambda} M_{\lambda} . M$ is PWP with respect to $E$ if and only if for each $\lambda \in \Lambda, M_{\lambda}$ is $P W P$ with respect to $E$.

(2) Let $M=\prod_{\lambda \in \Lambda} M_{\lambda} . M$ is PWP with respect to $E$ if and only if for each $\lambda \in \Lambda, M_{\lambda}$ is $P W P$ with respect to $E$.

Proof. (1) Assume $M$ is PWP with respect to $E$. If $m_{\lambda} e_{i} R e_{i} b=0$, where $m_{\lambda} \in M_{\lambda}, e_{i} \in E$, and $b \in$ $R$ then $\left(0, \ldots, m_{\lambda}, 0, \ldots, 0\right) e_{i} R e_{i} b=0$. Since $M$ is PWP, $\left(0, \ldots, m_{\lambda}, 0, \ldots, 0\right) e_{i}=0$ or $M e_{i} b=0$. This implies that $m_{\lambda} e_{i}=0$ or $M_{\lambda} e_{i} b=0$ which means for each $\lambda \in \Lambda, M_{\lambda}$ is PWP with respect to $E$. Conversely assume that for each $\lambda \in \Lambda, M_{\lambda}$ is PWP with respect to $E$, and $\left(m_{1}, \ldots, m_{n}, 0, \ldots\right) e_{i} R e_{i} b=0$. This implies that $m_{\lambda} e_{i} R e_{i} b=0$. Since $M_{\lambda}$ is PWP with respect to $E, m_{\lambda} e_{i}=0$ or $M e_{i} b=0$. Hence $\left(m_{1}, \ldots, m_{n}, 0, \ldots\right) e_{i}=0$ or $M e_{i} b=0$. Thus $M$ is PWP with respect to $E$.

(2) It can be seen by similar method as in part (1).

Corollary 10. Let $E=\left\{e_{1}, \ldots, e_{n}\right\}$ be a complete set of left triangulating idempotents of $R$, let $M$ be an $R$-module, and let $F$ be a free $R$-module.

(1) $R$ is quasi-Baer if and only if $F$ is a PWP module with respect to $E$.

(2) $M$ is $P W P$ with respect to $E$ if and only if $F \otimes_{R} M$ is $P W P$ with respect to $E$.

Proof. It follows by [1, Theorem 4.11] and Proposition 9.

Proposition 11. Let $M$ be an $R$-module, and $S=\operatorname{End}_{R}(M)$. Then ${ }_{S} M$ is prime if and only if $\tau \operatorname{dim}(S)=1$, and $M_{R}$ is quasiBaer.

Proof. $(\Rightarrow)$ Since $M$ is a prime $S$-module, then for each $N \subseteq$ $M$, l.ann ${ }_{S}(N)=1 \cdot \operatorname{ann}_{S}(M)=0$. This implies that $M_{R}$ is quasiBaer. If $e^{2}=e \in S$, then $M=e M \oplus(1-e) M$. Since ${ }_{S} M$ is prime, $1 . \operatorname{ann}_{S}(e M)=1 \cdot \operatorname{ann}_{S}((1-e) M)=1 \cdot \operatorname{ann}_{S}(M)$. This implies that $e=1$ or $e=0$. Thus $\tau \operatorname{dim}(S)=1$.
$(\Leftarrow)$ Let $N$ be any submodule of ${ }_{S} M$. Since $M_{R}$ is quasiBaer, $\operatorname{lann}_{S}(N)=S e$, where $e \in S_{r}(S)$. Since $\tau \operatorname{dim}(S)=1, e \in$ $\{0,1\}$. If $e=1$, then $N=0$. Thus $e=0$. This implies that for each nonzero submodule $N \subseteq_{S} M$, $\operatorname{lann}_{S}(N)=1 \cdot \operatorname{ann}_{S}(M)=$ 0 . This means ${ }_{S} M$ is prime.

It is folklore that prime radical plays an important role in the study of rings [7]. Following this concept is developed for modules of course by using a complete set of left triangulating idempotents of $R$.

Definition 12. Let $M$ be an $R$-module, let $N$ be a proper submodule of $M$, and let $E=\left\{e_{1}, \ldots, e_{n}\right\}$ be a complete set of left triangulating idempotents of $R$.

(1) The piecewise prime radical of $N$ in $M$ with respect to $E$ is denoted by $\operatorname{PRad}(N)$ and is defined to be the intersection of all piecewise prime submodules of $M$ with respect to $E$ containing $N$.

(2) $\operatorname{PRad}(M)$ means the intersection of all piecewise prime submodules of $M$ with respect to $E$. If $M$ has no piecewise prime submodule with respect to $E$, then $\operatorname{PRad}(M)=M$.

Proposition 13. Let $N$ be a submodule of $R$-module $M$.

(1) If $N$ is a submodule of $R$-module $M$, then $\operatorname{PRad}(N) \subseteq$ $\operatorname{PRad}(M)$.

(2) If $\operatorname{PRad}(M)=K$, then $\operatorname{PRad}(M / K)=0$.

(3) If $M=\oplus_{i \in I} M_{i}$ is a direct sum of submodules $M_{i}$, then

$$
\operatorname{PRad}(M)=\bigoplus_{i \in I} \operatorname{PRad}\left(M_{i}\right)
$$

Proof. Let $E=\left\{e_{1}, \ldots, e_{n}\right\}$ be a complete set of left triangulating idempotents of $R$.

(1) Let $K$ be any piecewise prime submodule of $M$ with respect to $E$. If $N \subseteq K$, then $\operatorname{PRad}(N) \subseteq K$. If $N \nsubseteq K$, then by the definition it is easy to see that $N \cap K$ is a piecewise prime submodule of $N$ with respect to $E$. Thus $\operatorname{PRad}(N) \subseteq(K \cap N) \subseteq K$. Hence $\operatorname{PRad}(N) \subseteq$ $\operatorname{PRad}(M)$.

(2) Let $P / K$ be a piecewise prime submodules of $M / K$ with respect to $E$. By definition $(M / K) /(P / K)$ is a piecewise prime module with respect to $E$. Thus $M / P$ is a piecewise prime module with respect to $E$. This implies that $P$ is a piecewise prime submodules of $M$ with respect to $E$. Hence $\operatorname{Pad}(M / K)=0$.

(3) By (1) for each $i \in I, \operatorname{PRad}\left(M_{i}\right) \subseteq \operatorname{PRad}(M)$. This implies that

$$
\bigoplus_{i \in I} \operatorname{PRad}\left(M_{i}\right) \subseteq \operatorname{PRad}(M)
$$

Let $\left(m_{i}\right)_{i \in I} \in M \backslash \oplus_{i \in I} \operatorname{PRad}\left(M_{i}\right)$. Then there exists $i \in I$, such that $m_{i} \notin \operatorname{PRad}\left(M_{i}\right)$. By the definition there exists a piecewise prime submodule $N_{i} \subseteq M_{i}$ with respect to $E$, such that $m_{i} \notin N_{i}$. If $K=N_{i} \oplus\left(\oplus_{i \neq j} M_{j}\right)$, then $K$ is a piecewise 
prime submodule of $M$ with respect to $E$, and $m \notin K$. Thus $m \notin \operatorname{PRad}(M)$. It means that

$$
\operatorname{PRad}(M)=\bigoplus_{i \in I} \operatorname{PRad}\left(M_{i}\right)
$$

\section{References}

[1] G. F. Birkenmeier, H. E. Heatherly, J. Y. Kim, and J. K. Park, "Triangular matrix representations," Journal of Algebra, vol. 230, no. 2, pp. 558-595, 2000.

[2] J. Dauns, "Prime modules," Journal für die Reine und Angewandte Mathematik, vol. 298, pp. 156-181, 1978.

[3] E. H. Feller and E. W. Swokowski, "Prime modules," Canadian Journal of Mathematics, vol. 17, pp. 1041-1052, 1965.

[4] M. Behboodi, O. A. S. Karamzadeh, and H. Koohy, "Modules whose certain submodules are prime," Vietnam Journal of Mathematics, vol. 32, no. 3, pp. 303-317, 2004.

[5] M. Behboodi and H. Koohy, "On minimal prime submodules," Far East Journal of Mathematical Sciences, vol. 6, no. 1, pp. 8388, 2002.

[6] S. T. Rizvi and C. S. Roman, "Baer and quasi-Baer modules," Communications in Algebra, vol. 32, no. 1, pp. 103-123, 2004.

[7] T. Y. Lam, A First Course in Noncommutative Rings, Springer, New York, NY, USA, 1991. 

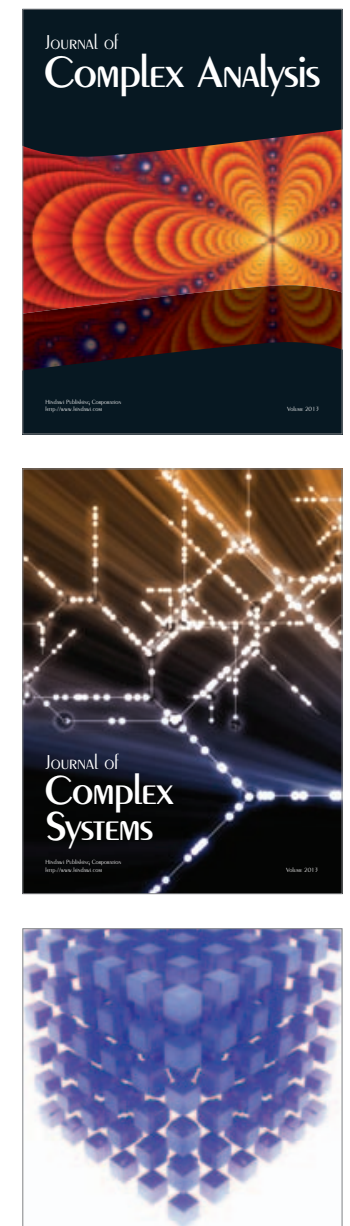

Mathematical Problems in ENGiNEERING
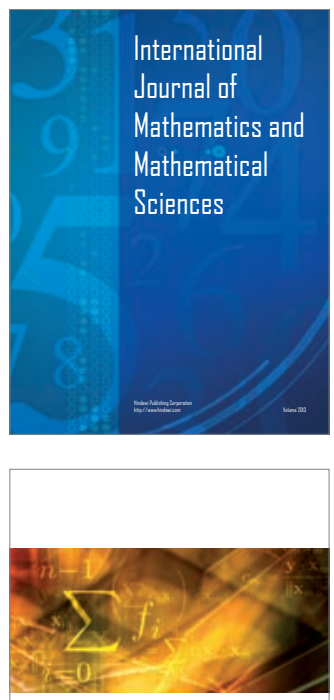

ISRN

Applied

Mathematics
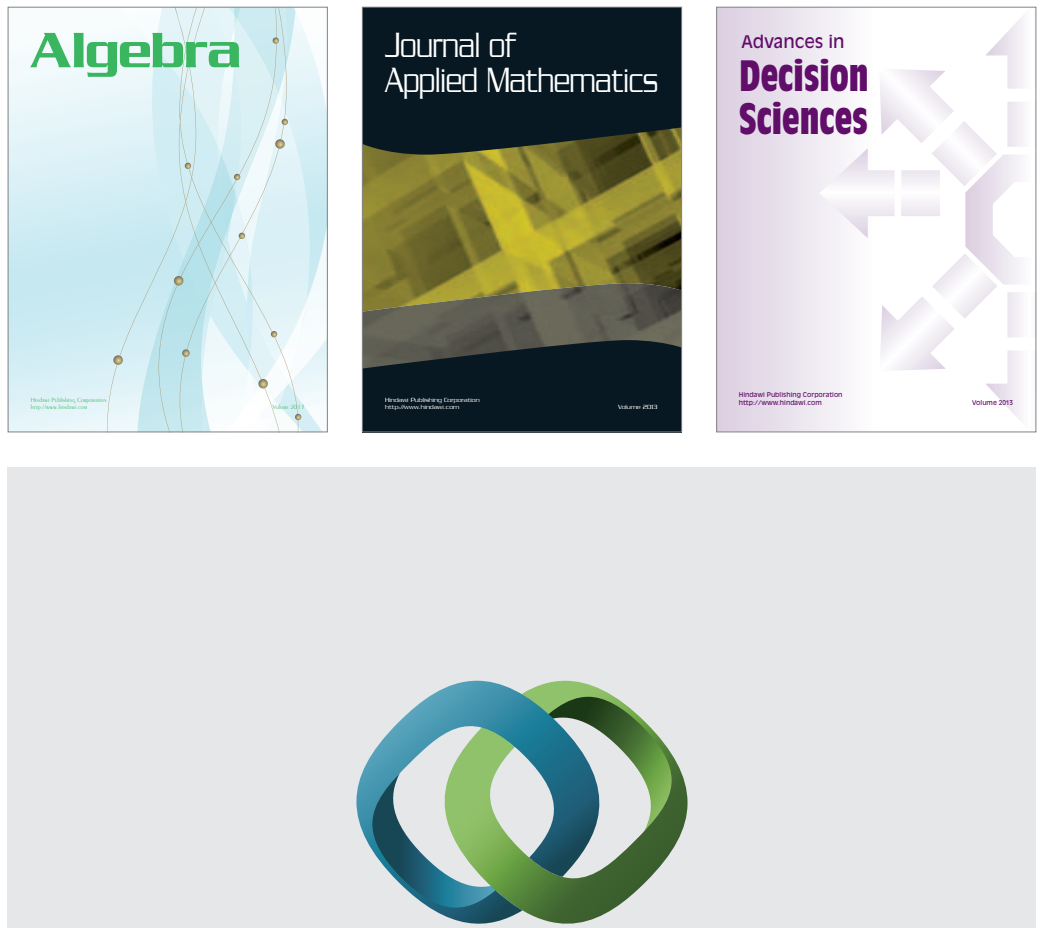

\section{Hindawi}

Submit your manuscripts at http://www.hindawi.com

Game Theory
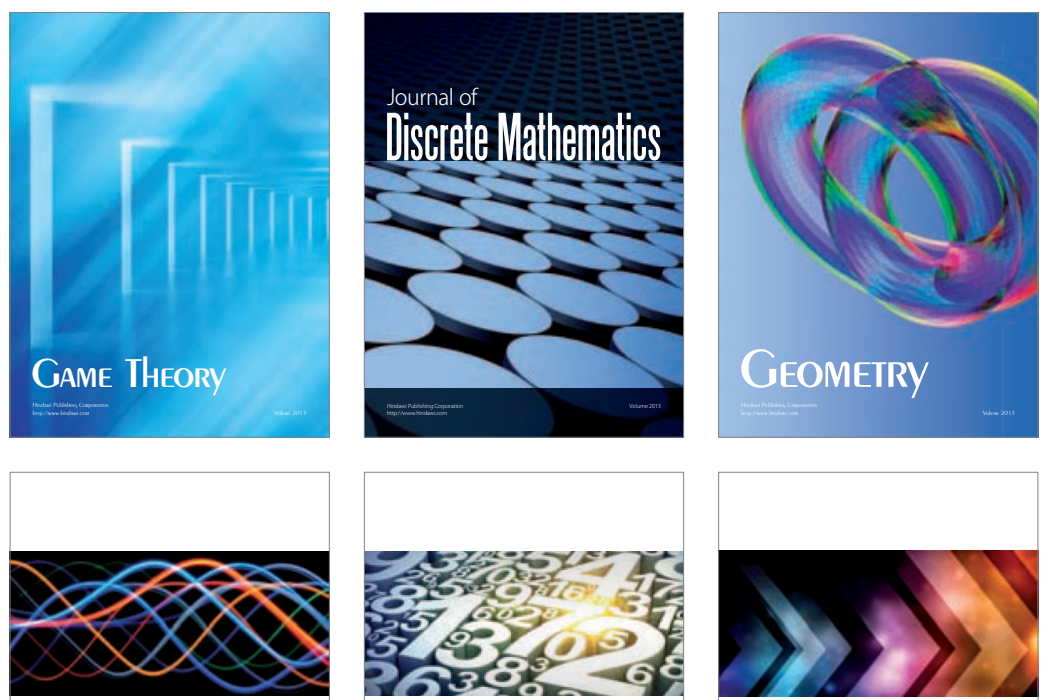

ISRN

Mathematical

Physics

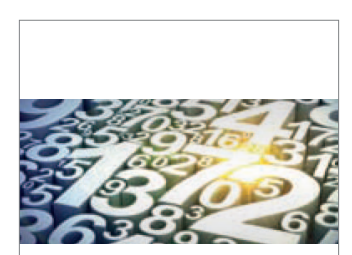

ISRN

Combinatorics

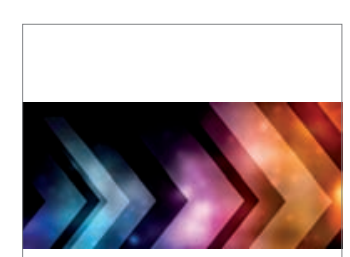

ISRN

Operations

Research
ISRN

Computational

Mathematics

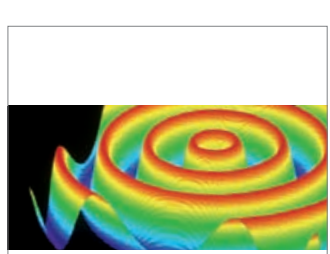

Abstract and

Applied Analysis
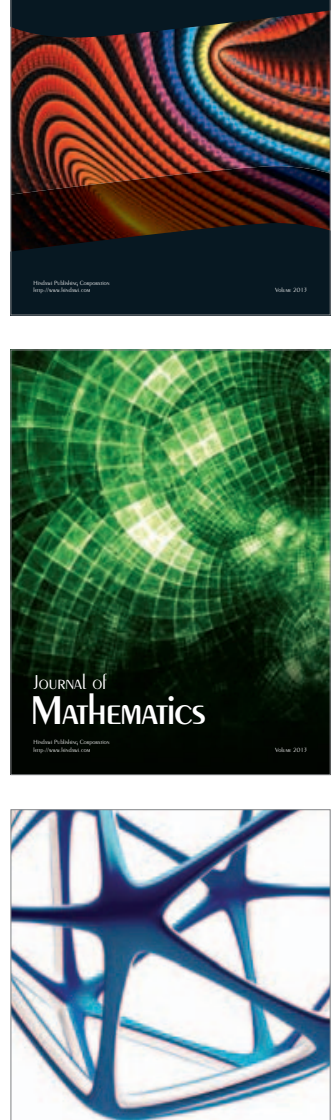

Ilownul of
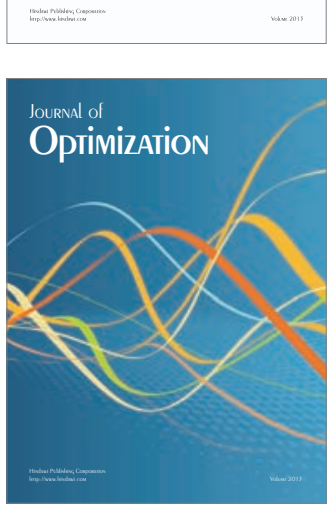

Mathematics 


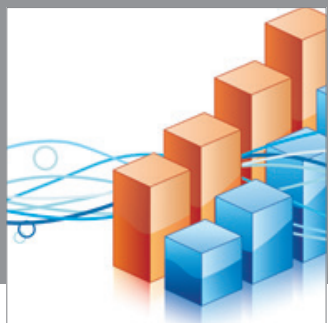

Advances in

Operations Research

mansans

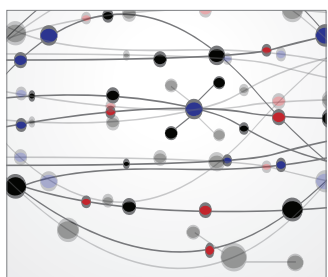

The Scientific World Journal
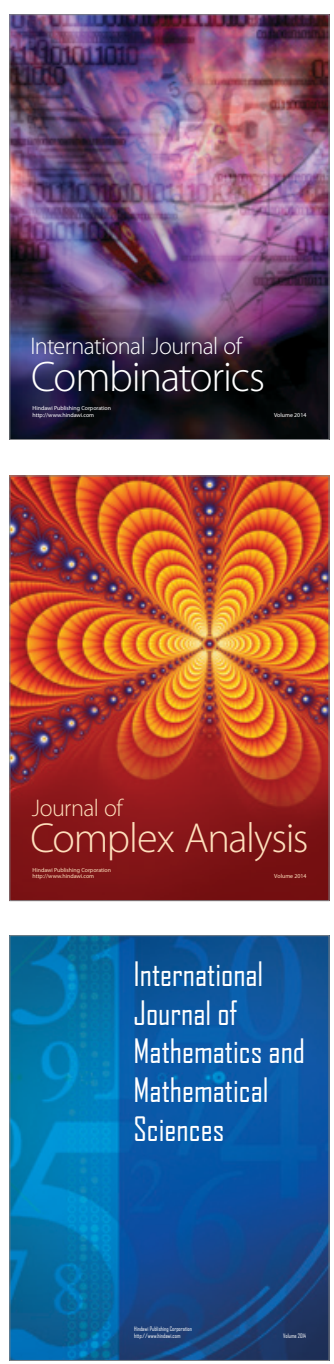
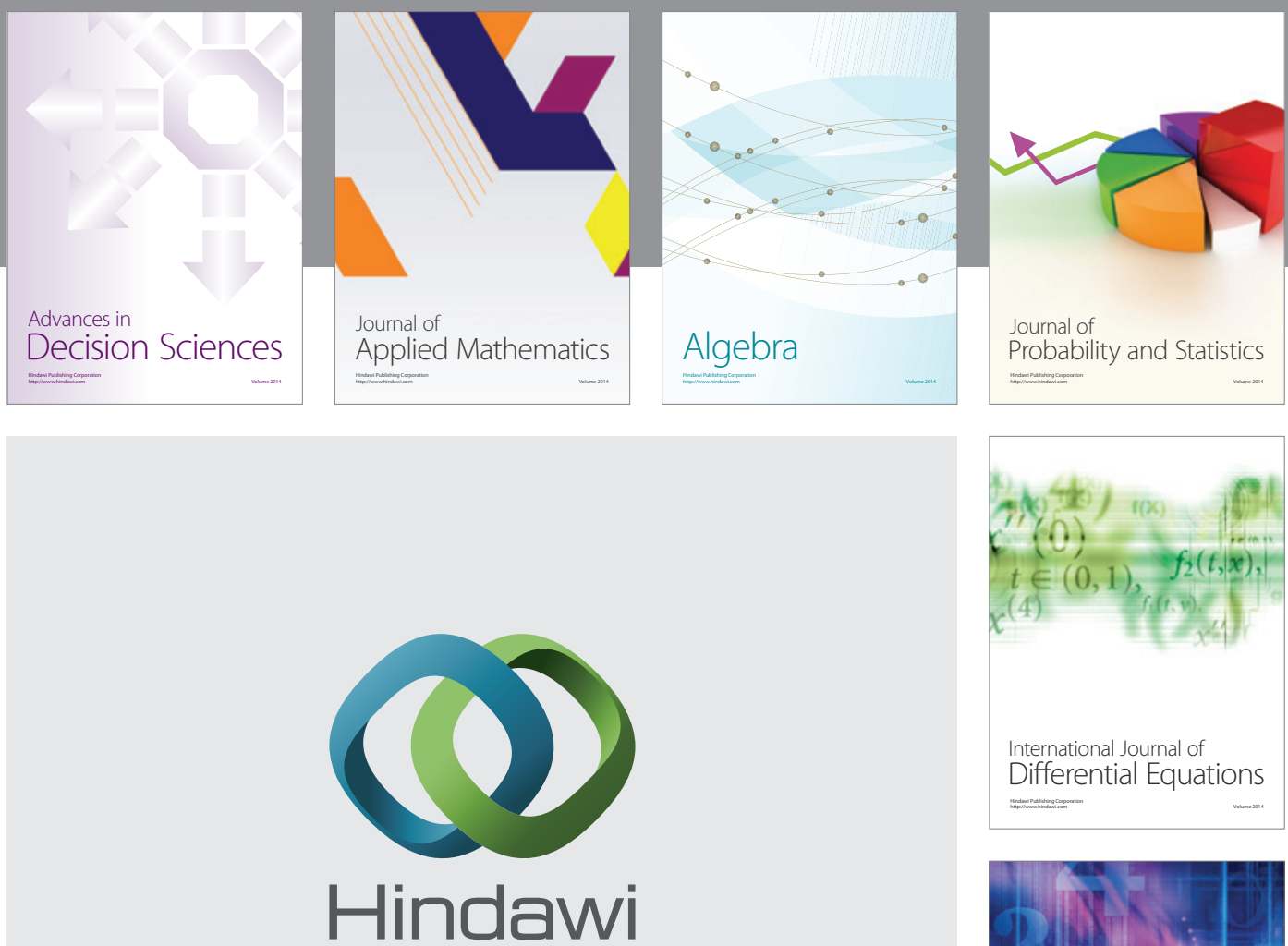

Submit your manuscripts at http://www.hindawi.com
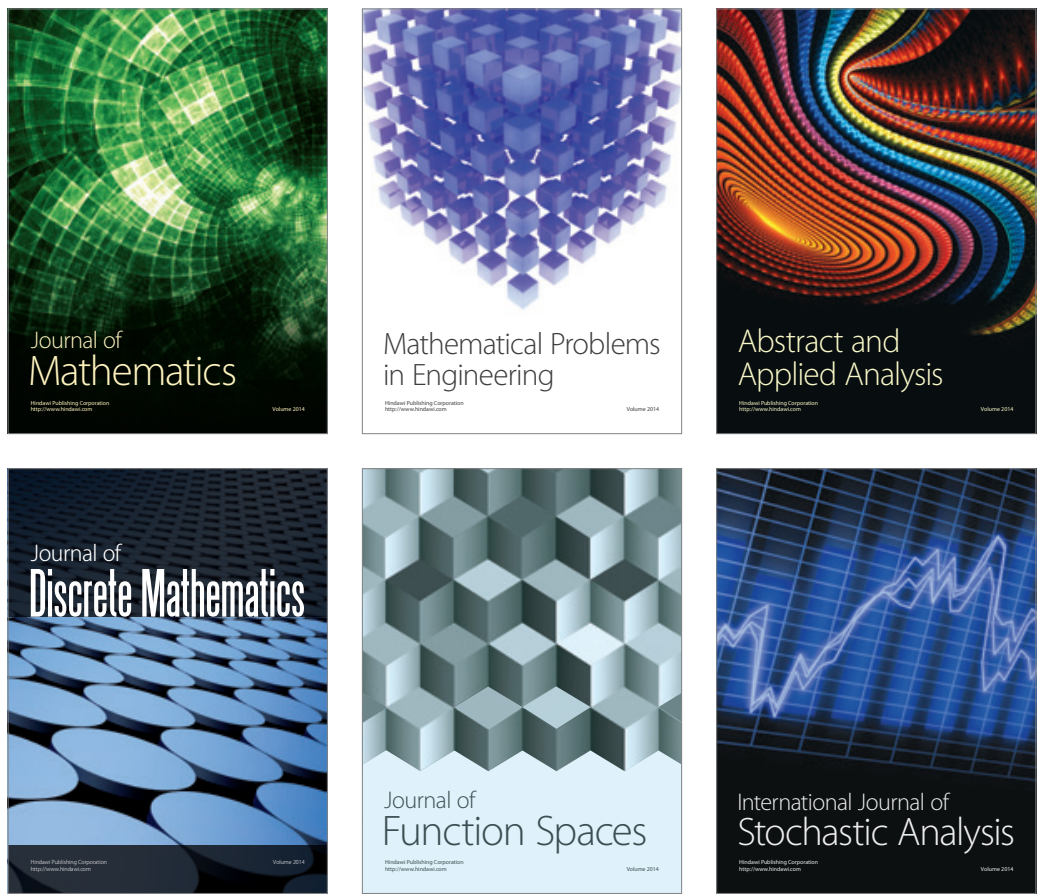

Journal of

Function Spaces

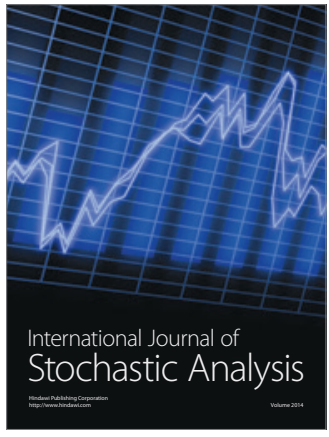

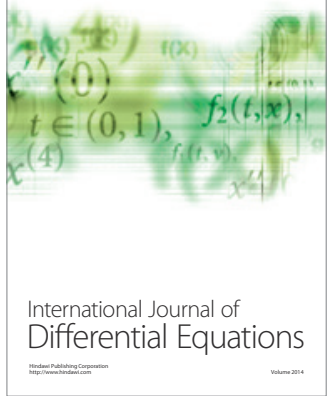
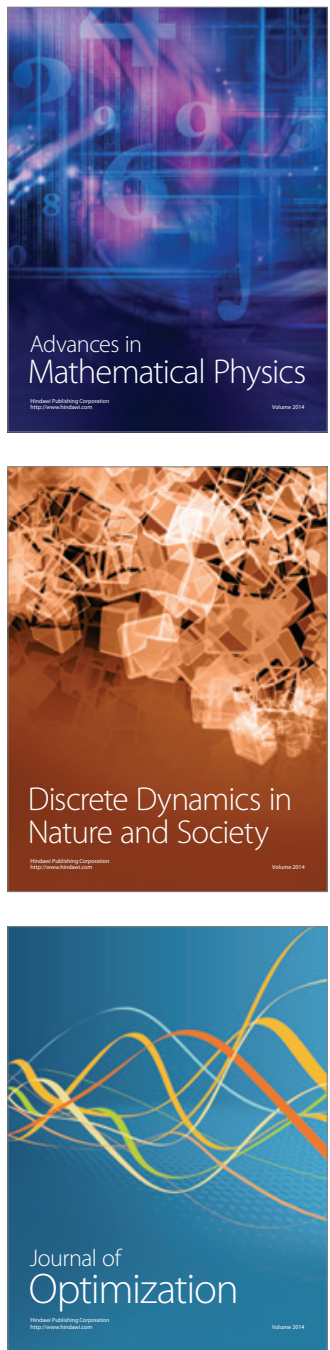